\title{
Phylogenetic, Molecular, and Functional Characterization of PpyCBF Proteins in Asian Pears (Pyrus pyrifolia)
}

\author{
Mudassar Ahmad 1,2,3,+, Jianzhao Li 1,2,3,+, Qinsong Yang 1,2,3, Wajeeha Jamil 1,2,3, \\ Yuanwen Teng ${ }^{1,2,3, *}$ and Songling Bai ${ }^{1,2,3, *(D)}$ \\ 1 Department of Horticulture, Zhejiang University, Hangzhou 310058, Zhejiang, China; \\ ahmad_mudassar@zju.edu.cn (M.A.); 21316043@zju.edu.cn (J.L.); qsyang@zju.edu.cn (Q.Y.); \\ 11616122@zju.edu.cn (W.J.) \\ 2 The Key Laboratory of Horticultural Plant Growth, Development and Quality Improvement, \\ the Ministry of Agriculture of China, Hangzhou 310058, Zhejiang, China \\ 3 Zhejiang Provincial Key Laboratory of Integrative Biology of Horticultural Plants, Hangzhou 310058, \\ Zhejiang, China \\ * Correspondence: ywteng@zju.edu.cn (Y.T.); songlingbai@zju.edu.cn (S.B.) \\ + These authors contributed equally to this work.
}

Received: 24 March 2019; Accepted: 24 April 2019; Published: 26 April 2019

\begin{abstract}
C-repeat binding factor/dehydration-responsive element (CBF/DRE) transcription factors (TFs) participate in a variety of adaptive mechanisms, and are involved in molecular signaling and abiotic stress tolerance in plants. In pear (Pyrus pyrifolia) and other rosaceous crops, the independent evolution of CBF subfamily members requires investigation to understand the possible divergent functions of these proteins. In this study, phylogenetic analysis divided six PpyCBFs from the Asian pear genome into three clades/subtypes, and collinearity and phylogenetic analyses suggested that PpyCBF3 was the mother CBF. All PpyCBFs were found to be highly expressed in response to low temperature, salt, drought, and abscisic acid (ABA) as well as bud endodormancy, similar to PpyCORs (PpyCOR47, PpyCOR15A, PpyRD29A, and PpyKIN). Transcript levels of clade II PpyCBFs during low temperature and ABA treatments were higher than those of clades I and III. Ectopic expression of PpyCBF2 and PpyCBF3 in Arabidopsis enhanced its tolerance against abiotic stresses, especially to low temperature in the first case and salt and drought stresses in the latter, and resulted in lower reactive oxygen species (ROS) and antioxidant gene activities compared with the wild type. The increased expression of endogenous ABA-dependent and -independent genes during normal conditions in PpyCBF2- and PpyCBF3-overexpressing Arabidopsis lines suggested that PpyCBFs were involved in both ABA-dependent and -independent pathways. All PpyCBFs, especially the mother CBF, had high transactivation activities with 6XCCGAC binding elements. Luciferase and $\mathrm{Y} 1 \mathrm{H}$ assays revealed the existence of phylogenetically and promoter-dependent conserved $C B F-C O R$ cascades in the pear. The presence of a previously identified CCGA binding site, combined with the results of mutagenesis of the CGACA binding site of the PpyCOR15A promoter, indicated that CGA was a core binding element of PpyCBFs. In conclusion, PpyCBF TFs might operate redundantly via both ABA-dependent and -independent pathways, and are strongly linked to abiotic stress signaling and responses in the Asian pear.
\end{abstract}

Keywords: asian pears; CBF; gene functions; CRT/DRE binding sites 


\section{Introduction}

C-repeat binding factors/dehydration-responsive elements (CBFs/DREs) constitute a subfamily of the Apetala1/ethylene responsive factor (AP1/ERF) family and are characterized by the presence of one AP2 domain [1] that contains 60-70 highly conserved amino acid residues [2]. All CBFs have CBF signature motifs (PKK/RPAGRxKFxETRHP and DSAWR) that distinguish these factors from other AP1/ERF members harboring an AP2 domain [3]. This CBF motif specifically binds to the dehydration-responsive/C repeat (DRE/CRT) element (CCGAC) of downstream genes to regulate their expressions [4]. CBFs have a well-known role in cold response and acclimation in both herbaceous [5] and woody [6] plants. Studies on the poplar (Populus trichocarpa), eucalyptus (Eucalyptus globulus), grape (Vitis vinifera), sweet cherry (Prunus avium), birch (Betula pendula), citrus (Citrus paradisi), and dwarf apple (Malus baccata), have revealed that the cold acclimation function of CBF is highly conserved in these woody plants $[7,8]$. Nevertheless, several recent studies have suggested that the multiple CBF paralogs that have evolved in plants might perform different functions [9]. In this aspect, (i) CBF paralogs can influence each other's expressions. In Arabidopsis, for example, AtCBF2 negatively regulates the expressions of $A t C B F 1$ and $A t C B F 3$ [10]. (ii) In addition, CBF paralogs have different tissue specificities and expression times following cold stress. For example, PtCBF2 and PtCBF4 in poplars were detected only in leaves, whereas PtCBF1 and PtCBF3 were also expressed in leaves, stems, and dormant buds [11]. A similar result has also been reported in grapes, where Vitis CBF4 was present in mature leaves and buds, while Vitis $C B F 1, C B F 2$, and $C B F 3$ were only found in young leaves and buds $[12,13]$. (iii) Several $C B F$ genes have also been found to be induced by other abiotic stresses (drought and salt) and molecular signals (such as abscisic acid signaling). These include GmDREB1G-1 and GmDREB1G-2 in soybeans [14], $\operatorname{VrCBF1}$ and $\operatorname{VrCBF} 4$ in grapse [9], MbDREB1A in dwarf apples [15], and AtDDF1, AtDDF2 [16], and AtCBF4 [17] in Arabidopsis. (iv) Overexpressed CBF paralogs from other species conferred various levels of abiotic stress tolerance on plants. For example, overexpression of both $\operatorname{VrCBF1}$ and $\operatorname{VrCBF} 4$ enhances abiotic stress tolerance in Arabidopsis, but $\operatorname{VrCBF1}$ is mainly responsible for drought tolerance, while $\operatorname{VrCBF} 4$ confers most of the cold tolerance [9].

A core set of robustly stress-responsive plant genes, known as COR (cold-regulated), $R D$ (responsive to dehydration), and KIN (cold-induced), have been identified from numerous differential screening and cloning studies over the years. Many COR genes contain one or more similar CRT (CCGAC) elements in their promoters, which are also found in CRT/DRE genes, and interestingly, they all have abiotic stress responsiveness [18]. Abiotic stress rapidly induces $C B F$ s, which then activate various downstream cold-responsive $(C O R)$ genes whose products collectively increase a plant's abiotic tolerance capacity through necessary physiological and biochemical alterations [19]. The cold-stress induction of $C B F$ and COR genes is also regulated by the circadian clock [20]. An important feature of abiotic stresses, especially low temperature, is a hyperosmotic signal that causes the phytohormone abscisic acid (ABA) to accumulate. ABA in turn provokes many adaptive responses, such as bud endodormancy, in plants [21]. Low temperatures and ABA have recently been reported to synergistically promote cold-hardiness and CBF expression in dormant grape buds [21]. These adaptive mechanisms are not only affected by ABA contents, but also by ABA signaling pathways [22]. For example, high ABA levels lead to endodormancy [23], inhibition of ABA pathways promotes germination and lateral root formation [24], while the reduction of ABA enhances water transpiration through stomatal pores [25].

Adaptive mechanisms, molecular signaling, and tolerance to abiotic stresses are also determined by many up- and downstream transcription factors of $C B F$ genes. During the adaptive process of bud endodormancy in pears, for example, P $P$ ICE3 works upstream of P $p C B F 1$, while P $p C B F 1, P p C B F 2$ and $P p C B F 4$ activate downstream $P p D A M 1$ and $P p D A M 3$ genes that induce endodormancy by inhibiting PpFT2. Meanwhile, microRNA miR6390 degrades dormancy associated MADS (DAM) box genes to release endodormancy [22,26]. MdMYB and MdHY5 in apples and PbeNAC1 in pear have also been found to be involved in the regulation of $C B F$ genes and the acquisition of abiotic stress tolerance [27-29]. In regards to molecular signals such as ABA, the PYR/RCAR-PP2C complex [30] inhibits PP2C [31] and activates SnRK2s, which not only target ABA-responsive genes (ABF/ABI5-type basic/region leucine 
zipper) [32], but also phosphorylate ICE1 to activate CBF-COR cascades and promote plant tolerance through ABA signaling [33]. During abiotic stress, many transcription factors, i.e., COLD1, NAC, bHLH, ICE1, MYB, SnrK2, ABF, HOS1, and SIZ1, have been found to function upstream of CBFs, while $\mathrm{ADF}, \mathrm{ZAT}, \mathrm{LOS}, \mathrm{SFR}$, and RAP function downstream to induce plant tolerance [34]. Consequently, $\mathrm{CBF}$ is the central regulator of plant adaptation and abiotic stress tolerance via both ABA-dependent and -independent pathways [15].

Pyrus germplasm resources, which are distributed worldwide, are most plentiful in China, especially in the western and southwestern mountainous areas $[35,36]$. Numerous genes and TFs with functions related to plant dispersal, adaptation to natural habitats, and stress tolerance had been identified and characterized in plants, including AREB/ABF, MYB, AP2/EREBP, bZIP, HSF, CBF/DREB, MYC, HB, NAC, and WRKY. Among them, the CBF/DREB subfamily occupies a major position in both herbaceous [5] and woody [6] plants. The complete CBF subfamily and the possible divergent functions of its members have never been fully studied in rosaceous groups. In this study, we identified 15 PpyCBFs from the pear genome database, but were unable to predict their functions through phylogenetic analysis. Hence, we tested the hypothesis to know whether all PpyCBF paralogs had different functions or not. We therefore selected six of the 15 PpyCBFs after characterization and checked their responses to abiotic stresses, ABA treatment, and bud endodormancy compared with abiotic stress-responsive PpyCOR genes. We also generated PpyCBF2- and PpyCBF3-overexpressing Arabidopsis plants and analyzed their abiotic stress tolerances, endogenous gene expressions, and ROS accumulations. After checking the binding activity of all PpyCBFs with the cis-element (CCGAC), we also studied their possible abiotic regulatory pathways and binding sites in pears.

\section{Results}

\subsection{Identifications and Characterizations of PpyCBF Subfamily}

To identify PpyCBFs, we first carried out a hidden Markov model search against the pear genome database. This approach identified 15 PpyCBF TFs, which were then subjected to phylogenetic analysis and further confirmation of their sequence identities and chromosomal positions. Pairwise sequence identities among isolated PpyCBFs were all very high, ranging from 0.271 (PpyCBF9 and PpyCBF10 vs. PpyCBF12) to 0.994 (PpyCBF15 vs. PpyCBF4) (Table S1). Sequences that had an identity $>0.90$ and were on the same phylogenetic branch (PpyCBFs 7,8,9,10,11,12,13,14), incomplete (PPyCBF12), or on a scaffold (PpyCBFs 7,8,10,11,13,14,15) were eliminated from further analysis, whereas their corresponding sequences, i.e., PpyCBFs 1-6, were retained (Figure 1a, Table S1). To explore evolutionary relationships within the isolated subfamily, we first constructed a phylogenetic tree of sequences of similar candidates in Pyrus (Ppy), Arabidopsis (At), Malus (Md), Prunus (Ppe), Fragaria (Fv), and Vitis $(V v)$. The phylogenetic analysis distributed the PpyCBFs into three main clades/subtypes: PpyCBF3 in clade I, PpyCBFs 1,2,4 in clade II, and PpyCBF5 and PpyCBF6 in clade III. Interestingly, PpyCBFs, along with $C B F s$ of other rosaceous crop species, appeared to be evolved independently of model crop CBFs (AtCBFs 1-4). With the exception of PpyCBF3, which was clustered in clade I with Arabidopsis $C B F s$, all other PpyCBFs were placed in clades II and III with MdDREBs and PpeDREBs (Figure 1a). This independent evolution of $P p y C B F s$ suggested their potential divergent functions and served as the impetus for our study to explore and elucidate the regulation of this family in pears.

Since PpyCBFs belong to the AP2/ERF family, we performed a collinearity analysis of the entire family to understand $P p y C B F$ evolution and gene duplication (Figure S1a). We found 68 duplicated AP2/ERF pairs. Among them, two pairs, i.e., Pbr013924(PpyCBF3):Pbr032764(PpyCBF5) and Pbr013924(PpyCBF3):Pbr021781(PpyCBF1), belonged to its PpyCBF subfamily (Figure S1b). These results suggest that clades II and III of CBFs, i.e., PpyCBF1 and PpyCBF5, evolved from PpyCBF3, which was found in an ancestral clade with both monocot and dicot plants (Figure 1a). To examine diversification in gene structures and uncover potential conserved motifs in these selected PPyCBFs, we constructed another phylogenetic tree, which revealed that both duplicated PpyCBF3 and PpyCBF5, 
and PpyCBF2 and PpyCBF4 had potentially similar functions. In addition, PpyCBF5 together with PpyCBF6 were in a sister relationship with a cluster comprising PpyCBF1 and PpyCBFs 2,4, with the branch leading to these genes in turn joined to the ancestral $C B F$ (Figure 1b). Regarding gene structures and conserved motifs, PpyCBF5 was the only gene with just one intron. All the others had exonic regions (Figure 1c). Alignment of PpyCBFs in each phylogenetic clade revealed 10 different types of common motifs (Figure 1d). These findings indicate that PpyCBFs in the same clade have similar gene structures and motifs, and possibly similar functions.

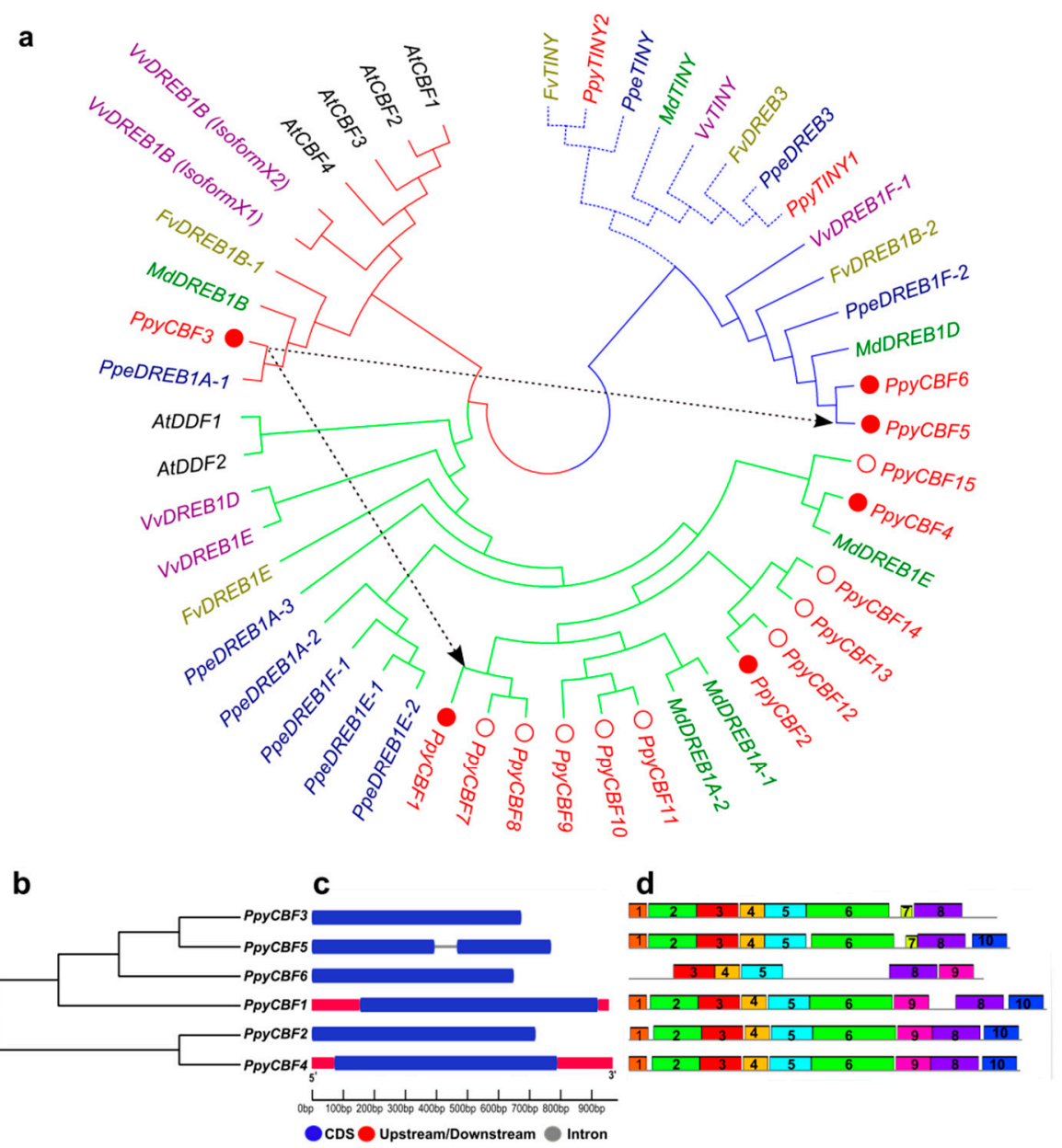

Figure 1. Identification and characterization of PpyCBFs. (a) Phylogenetic analysis of PpyCBF transcription factors with similar TFs of Arabidopsis (At), Malus (Md), Prunus (Ppe), Fragaria (Fv), and Vitis $(V v)$ species. Red, green, and blue colors indicate clades/subtypes I, II, and III of CBFs, respectively, while compact and hollow red circles indicate selected and rejected PpyCBFs, respectively. Arrow lines indicate the evolution of clades II and III from clade I. (b) Phylogenetic analysis of selected PpyCBFs. (c) Gene structure of PpyCBFs. Blue, black, and red lines indicate exon, intron, and upstream/downstream sections in gene structure. (d) Protein motif: Schematic diagrams of possible conserved motifs (1-10) in PpyCBF proteins, indicated by different colors.

\subsection{Strong Induction of PpyCBF Transcription by Various Abiotic Stresses and ABA Treatment}

To better understand the functions of PpyCBFs, we examined transcript levels of PpyCBFs in explants of Pyrus pyrifolia 'Dangshan Suli' subjected to different abiotic stress treatments, i.e., low temperature $\left(4^{\circ} \mathrm{C}\right)$, drought $(15 \%$ polyethylene glycol (PEG)) and salt $(200 \mathrm{mM} \mathrm{NaCl})$, for $0,6,12,24$, and $48 \mathrm{~h}$. qRT-PCR analysis revealed that the expressions of all six PpyCBF genes were induced by all abiotic stresses, but each gene responded differently to various stresses depending on its associated 
clade (Figure 2a). During cold treatment, expressions of PpyCBFs were all constant from 6 to $48 \mathrm{~h}$ and significantly higher than the control, with relative abundances of clade II CBFs which were much higher ( 200-1600) than those of clade I and II CBFs ( 2-50). During salt treatment, all PpyCBFs were statistically at their maximums after 12 and $48 \mathrm{~h}$ except for PpyCBF4 (which peaked only at $48 \mathrm{~h}$ ). The responses of clade I and III PpyCBFs were higher at early stages of salt stress than those of clade II PpyCBFs. Under drought conditions, PpyCBF3 (12 h), PpyCBF2 (24 h), PpyCBF4 (24 h), and PpyCBF5 (48 h) were accentuated, while PpyCBF1 and PPyCBF6 were downregulated. To determine whether PpyCBFs respond to ABA, we also tested their expressions in pear calli after 0, 3, 6, 12, and $48 \mathrm{~h}$ of ABA treatment $(100 \mu \mathrm{M})$. Notably, all PpyCBFs had responses to ABA after 3 and $48 \mathrm{~h}$. Short-term ABA exposure significantly promoted the expressions of clade II PpyCBFs, whereas longer exposure significantly induced the members of the other two clades (PpyCBF3 and PpyCBF6). Expression levels of clade II PpyCBFs were much higher than those of clades I and III. Significant downregulation of PpyCBF3 (24 h), PpyCBF1 (24 h), PpyCBF5 (6 h), and PpyCBF6 (12 h) was also observed during ABA treatment of pear calli (Figure 2a). In summary, clade I and III PpyCBFs exhibited higher levels of transcripts during salinity and drought treatments, whereas clade II PpyCBF transcripts were more abundant during low temperature and ABA stresses.

We also compared the expressions of PpyCBFs with those of COR genes (PpyCOR47, PpyCOR15A, PpyRD29A, and PpyKIN) during ABA treatment and abiotic stress. qRT-PCR analysis uncovered highly significant expressions of PpyCORs during cold, salt, and drought stresses, the exception being PpyRD29A during drought. Likewise, PpyCORs exhibited a highly significant, constant response throughout ABA treatment (Figure $2 b$ ). To confirm the above results and check the stress status of explants and calli, we measured expression levels of antioxidant genes (PpySOD, PpyPOD, PpyAPX, and PpyCAT) during abiotic stress and those of ABA-responsive genes (PpyCYP707A-2, PpySnRK2-1 and PpySnRK2-4, PpyABi5, and PpyPYL-2) subjected to ABA treatment (Figure S2). The expressions of all these genes were found to be high. These results not only verify the effectiveness of the treatments, but also suggested that all PpyCBFs were differentially induced according to their clades during abiotic stresses and ABA treatments.

To understand the possible transcriptional regulatory cascades of $P p y C B F$, we also analyzed their promoters. We detected numerous cis elements responsive to biotic and abiotic stresses, molecular signaling, and plant adaptation in promoters of $P p y C B F$ transcription factors related to cold, salt, drought, oxidation, light, heavy metals, pathogens, heat, ABA, giberllic acid, and auxin, namely, ABI3/VP1, AP2/EREBP, AP2/RAV, ARF, bHLH, bZIP, ERF, GATA, MADS, MYB, MYC, NAC, TCP/PCF1, and WRKY cis elements (Table 1 and Table S2). We found varying degrees of differences between the types and numbers of $P p y C B F$ regulatory elements. The presence of these cis elements suggests that $\mathrm{ABA}$ and stress-inducible expressions of $P p y C B F s$ are transcriptionally regulated.

\subsection{Increased Transcripts of PpyCBFs Induced by Low Temperature and ABA during Pear Bud Endodormancy}

As inferred from the above results, all PpyCBFs responded to ABA and low temperature, two basic factors for the establishment of bud endodormancy. We therefore also verified the expressions of PpyCBFs during the endodormancy period from September to February in Asian pear cultivars 'Dangshan Suli' and 'Cuiguan' at 15-day intervals in 2016-2017 and 2017-2018. During bud endodormancy, we observed two peaks in PpyCBF expression, the first one related to low temperature and the other dependent on ABA. In both pear cultivars, all PpyCBFs had their first expression peaks on January 1-12, 2017, and January 10-11, 2018, with their maximum expressions on November 15 and October 15 of the two respective years (Figure 3). As reported in our previous study [22], below-normal maximum and minimum temperatures were observed from October 15 to November 15 during 2016-2017, with the winter season also delayed in 2016-2017 compared with 2017-2018 (November vs. October). These events ultimately affected the transcription of CBFs during both years. Nevertheless, PpyCBF transcripts in both cultivars had their second expression peaks between January 1-20, 2017, and from December 1, 2017, to January 1, 2018, with maximums observed 
in the middle of January and December in the two successive years. This indicated ABA-dependent responses of PpyCBFs during bud endodormancy (Figure 3) because, in our previous study of ABA-responsive genes, PpyNCED1,PpyCYP707A-3 and PpyCYP707A-4, and PpyLs 2,3,6,7,8 were at their peaks on January 1-20 during bud endodormancy [23]. Interestingly, the relative abundances of clade II PpyCBFs during low temperature and ABA peaks were higher than those of clades I and III during both years in both cultivars, consistent with our results discussed earlier (Figure 2a).
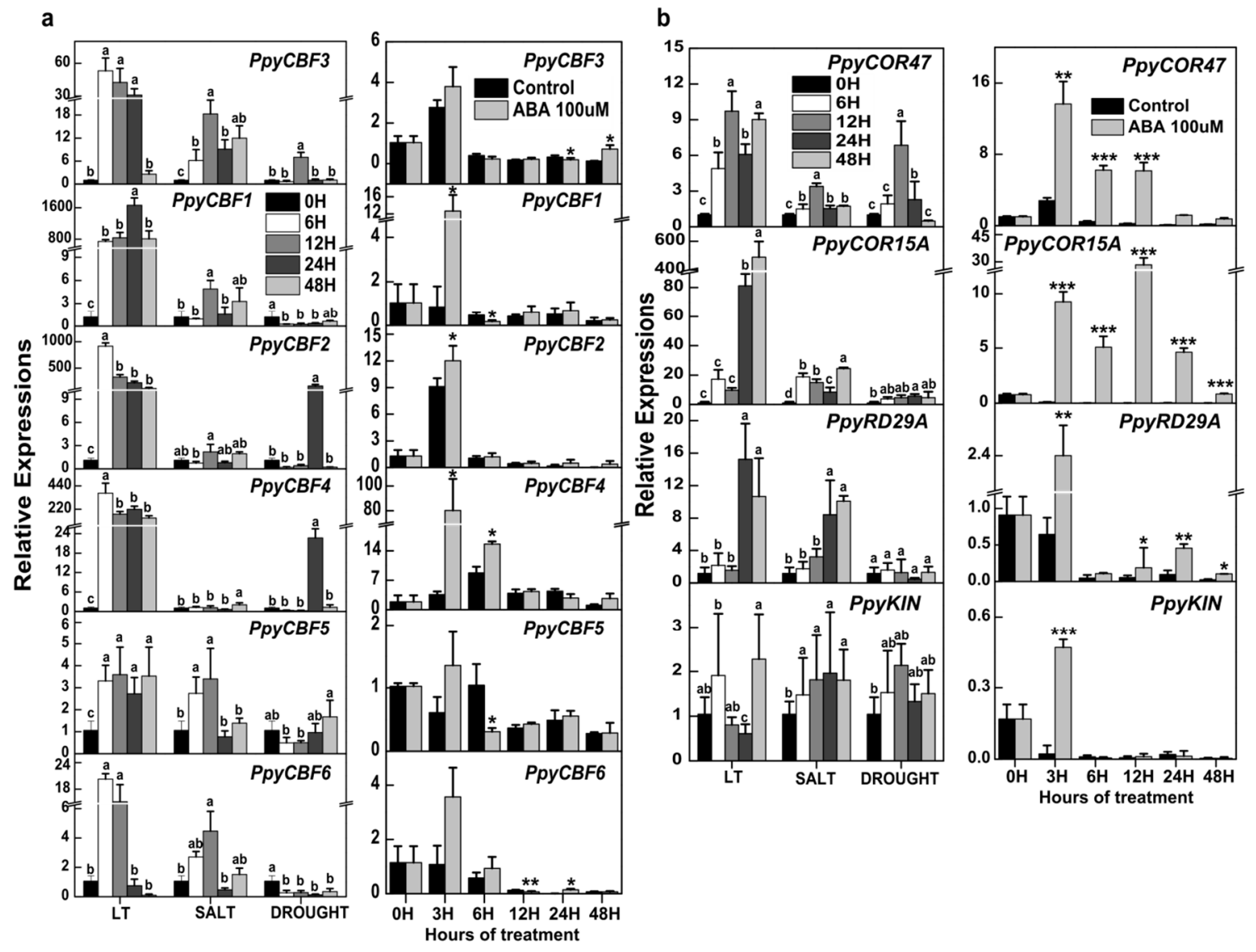

Figure 2. Relative expressions of PpyCBFs and PpyCORs during abiotic stresses and exogenous abscisic acid (ABA). (a) Expression analysis of $P p y C B F s$ during abiotic stresses (cold, salt, and drought) and ABA according to their phylogenetic clades. (b) Expression analysis of PpyCOR47, 15A, RD29A, and KIN in the same samples for comparison study. Both relative expressions were normalized to PpyActin expression level. Error bars indicate standard errors from three biological replicates $\left({ }^{*} p<0.05\right.$, $\left.{ }^{* *} p<0.01,{ }^{* * *} p<0.001\right)$ while means with different letters had significant differences $(p<0.05)$.

To further clarify low-temperature and ABA responses of PpyCBFs during bud endodormancy, we rechecked the responses of the studied PpyCORs during pear bud endodormancy to verify their high expressions during low temperature and ABA treatments (Figure $2 b$ ). Similar to the PpyCBFs, all PpyCORs (PpyCOR47, 15A, RD29A, and KIN) had expression peaks from November 15, 2016, to December 1, 2016, and from October 1, 2017, to November 1, 2017, corresponding to a low-temperature response, and from January 1-10, 2017, and from December 12, 2017, to January 1, 2018, corresponding to an ABA response, in both cultivars, with the exception of PpyKIN during 2016-2017 (Figure S3). The relative abundance of PpyCOR15A during low temperature and ABA peaks was higher than that of other CORs during low-temperature and ABA treatments (Figure 2b). These results not only reveal the responses of PpyCBFs and PpyCORs during bud endodormancy but also demonstrate their obvious correlation to each other. 
Table 1. Promoter analysis of all isolated PpyCBFs.

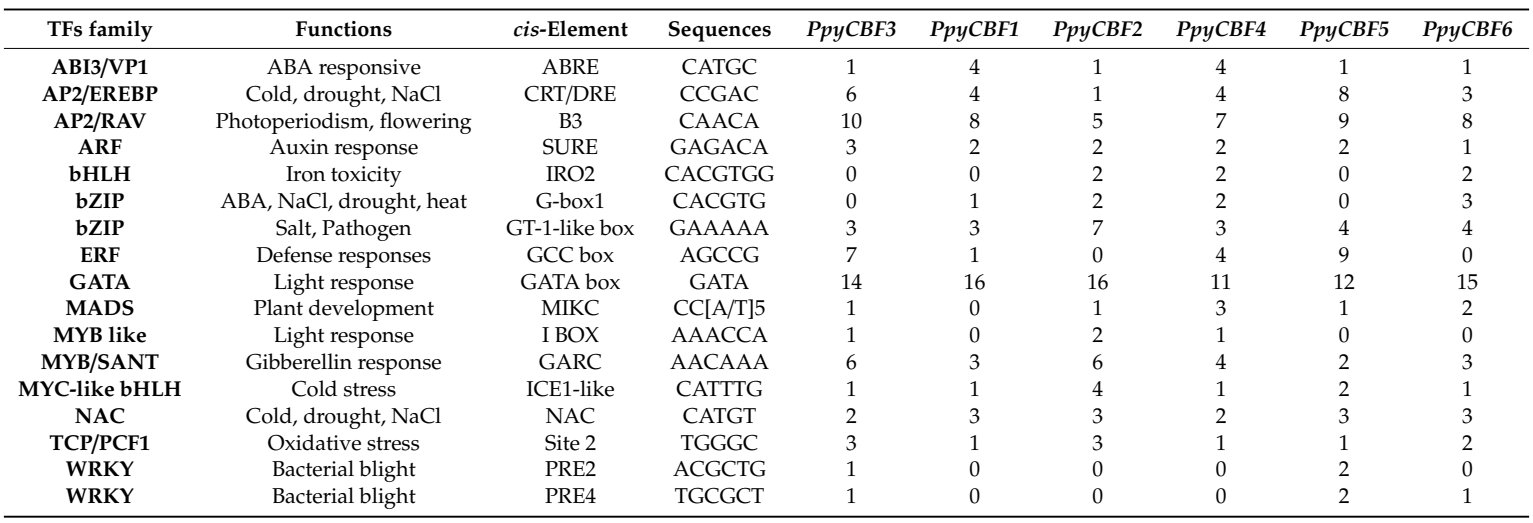
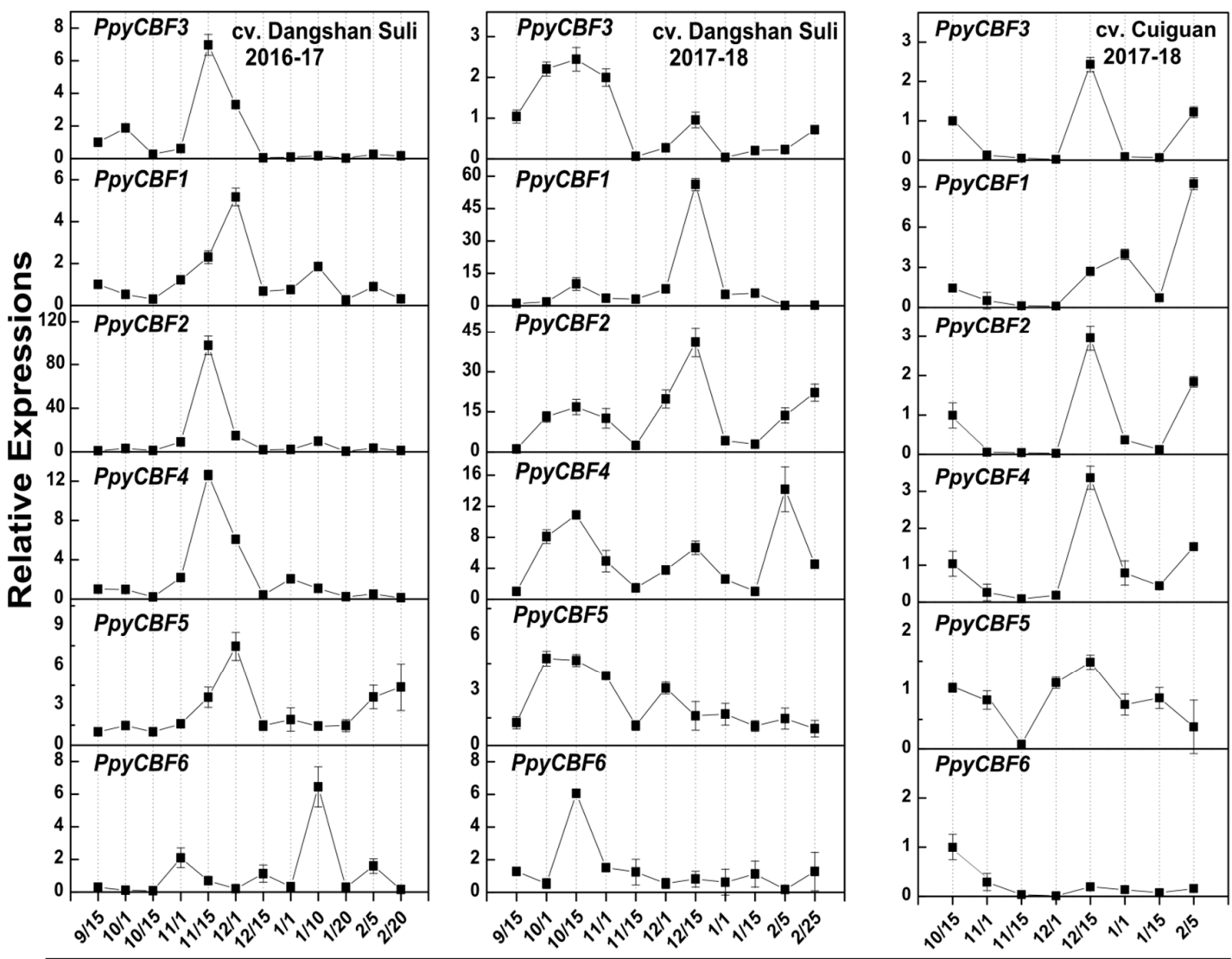

Bud Collection Dates

Figure 3. Relative expressions of PpyCBFs during bud endodormancy in Pyrus pyrifolia cv. 'Dangshan Suli' and 'Cuiguan' during two successive years 2016-2017 and 2017-2018. Buds were collected from September 15 to February 25 with about 15-day intervals. The data were normalized to PpyActin levels and the mean expression value was premeditated from four independent replicates. The standard deviation was shown by vertical bars.

\subsection{Overexpressions of PpyCBF2 and PpyCBF3 Positively Regulate Abiotic Stress Tolerances in} Transgenic Arabidopsis

To test whether PpyCBFs overexpression positively enhances abiotic stress tolerance, pCAMBIA1301 overexpression constructs of PPyCBF2 (the most transcriptionally activated CBF) and PpyCBF3 (the mother CBF) were transformed into Arabidopsis. Consistent with abiotic stress assays, phenotypes of both PpyCBF2-ox and PpyCBF3-ox transgenic lines were superior in several 
respects to the wild type (Figure S4a). Ectopic expression of PpyCBF2 and PpyCBF3 led to highly significantly increased root lengths after treatment with low temperature $(1.7$ and $1.3 \mathrm{~cm}$, respectively), salt $(1.5$ and $2.1 \mathrm{~cm})$, and drought $(2.0$ and $2.5 \mathrm{~cm})$ compared with wild-type plants $(0.8,0.7$, and $0.6 \mathrm{~cm}$ under low temperature, salinity, and drought, respectively), whereas no differences were observed among wild-type, PpyCBF2-ox, and PpyCBF3-ox plants under non-stress conditions (2.1, 2.2, and $1.9 \mathrm{~cm}$, respectively) (Figure 4a). Interestingly, PpyCBF2-ox plants under low temperature stress and PpyCBF3-ox plants under salinity and drought stress had more pronounced length increases relative to the wild type, but more growth retardation was observed in all plants during low temperature stress than during salt and drought stress.

a

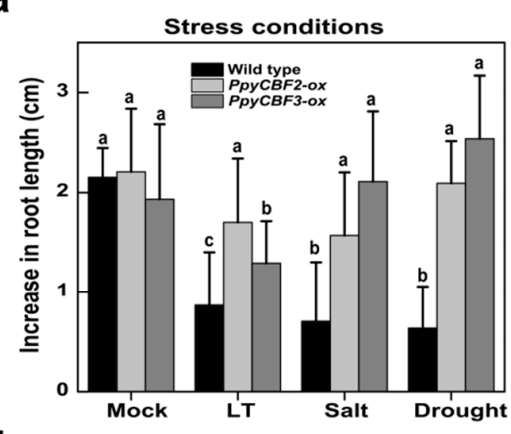

f

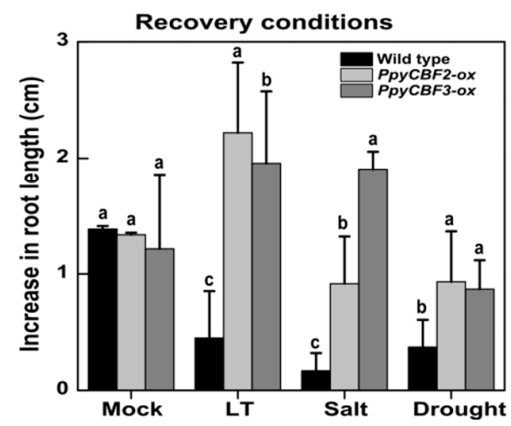

d

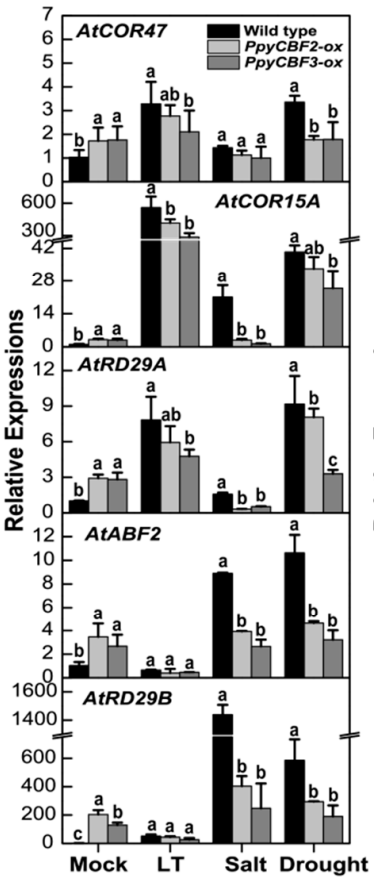

e

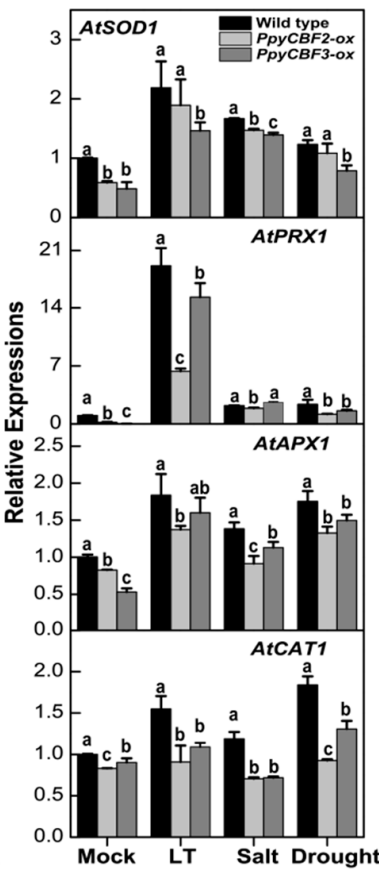

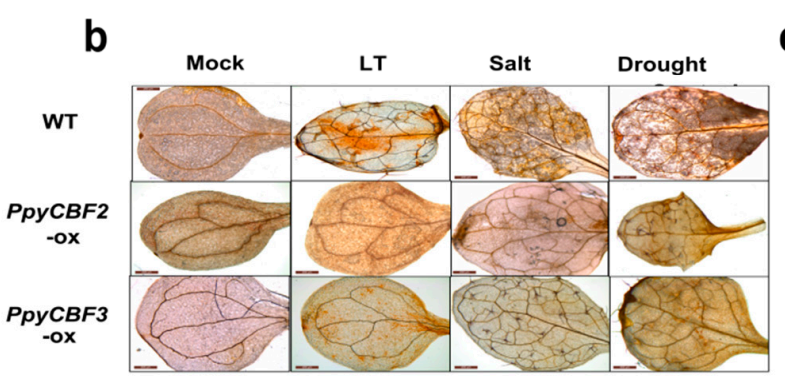

C Mock LT

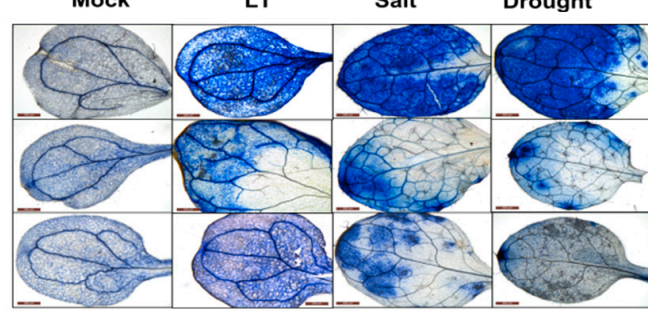

Figure 4. Overexpression analysis of PpyCBFs 2 and 3 in Arabidopsis during abiotic stresses. (a) Increase in root length (cm) of wild type (WT) and overexpressed lines during low temperature (LT), salt, and drought treatments by using ImageJ software. Error bars indicate standard errors from three biological replicates. (b,c) Diaminobenzidine (DAB) and nitroblue tetrazolium (NBT) staining of WT and overexpressed leaves after abiotic stresses to check ROS accumulation where brown and blue spots indicate the presence of $\mathrm{H}_{2} \mathrm{O}_{2}$ and $\mathrm{O}^{\cdot-}$ in situ while the red bar scale represent $200 \mu \mathrm{m}$. (d,e) Endogenous gene expressions of ABA-independent (AtCOR47, AtCOR15A and AtRD29A), ABA-dependent (AtABF2 and AtRD29B) and antioxidant genes (AtSOD1, AtPRX1, AtAPX1 and AtCAT1) in WT and overexpressed lines during control and abiotic stresses, normalized to AtPP2A expression levels. (f) Increase in root length to monitor the recovery among overexpressed and WTs Arabidopsis under normal conditions after abiotic stresses. Error bars indicate standard error from three biological replicates. Means with different letters had significant differences $(p<0.05)$. 
To confirm the effect of PpyCBF2-ox and PpyCBF3-ox on endogenous Arabidopsis genes, we examined the expressions of three ABA-independent (AtCOR47/RD17, AtCOR15a, and AtRD29A/COR78/LTI78), two ABA-dependent (AtABF2 and AtRD29B) and four antioxidant (AtSOD1, AtPRX1, AtAPX1, AtCAT1) genes. In Arabidopsis overexpressing either PpyCBF2 or PpyCBF3 under control or unstressed conditions, the ABA-dependent and -independent genes were significantly upregulated, and the antioxidant genes were downregulated (Figure $4 \mathrm{~d}, \mathrm{e}$ ). Under each stress treatment, relative abundances of all stress-responsive and antioxidant genes were significantly lower in both overexpressing Arabidopsis lines, relative to the wild type (Figure 4e), while antioxidant gene expressions were higher in PpyCBF3-ox plants than in PpyCBF2-ox ones. To verify the above results, we investigated the accumulations of $\mathrm{H}_{2} \mathrm{O}_{2}$ and $\mathrm{O}_{2}{ }^{\bullet-}$ by examining diaminobenzidine (DAB) and nitroblue tetrazolium (NBT) precipitation in PpyCBF2-ox, PpyCBF3-ox, and wild-type plants. Although no differences were apparent between wild-type and overexpressing plants under control conditions, more intense brown and blue precipitates were observed under abiotic stress in leaves of wild-type plants stained with DAB and NBT, respectively.

The results of DAB and NBT staining indicate that overexpressing plants accumulated less $\mathrm{H}_{2} \mathrm{O}_{2}$ and $\mathrm{O}_{2}{ }^{\bullet-}$ during abiotic stress than the wild type (Figure $4 \mathrm{~b}, \mathrm{c}$ ). The more pronounced activity of major $\mathrm{H}_{2} \mathrm{O}_{2}$ - and $\mathrm{O}^{\bullet-}$-scavenging enzymes (AtPRX, AtAPX, AtCAT and AtSOD) in wild-type plants was due to the higher accumulation of these toxic molecules, whereas the higher activity of antioxidant genes in PpyCBF3-ox plants indicated that scavenging of accumulated ROS was more successful in PpyCBF3-ox than in PpyCBF2-ox plants (Figure 4b,c,e).

After abiotic stress treatments, both wild-type and overexpressing plants were grown under control conditions for 7 days to monitor their recovery. Almost all CBF transgenic plants exhibited more pronounced prostrate growth during recovery than wild-type ones, which were found to be under severe stress (Figure S4b). After salt stress, both overexpressing lines experienced significant growth. Following low-temperature and drought treatments, PpyCBF2-ox and PpyCBF3-ox plants had significantly longer roots than their respective wild type (Figure 4f).

\subsection{PpyCBF Transcriptional Activation of 6X C-Repeat Binding Sites and Stress-Responsive Genes}

To examine $P P y C B F$ abiotic regulatory cascades, we first measured the CRT-dependent transactivation activities of $P p y C B F s$ in dual luciferase assays. For this analysis, full-length PpyCBFs were inserted into a SK vector, and 6X C-repeat binding sites (CCGAC) were inserted along with a $35 S$ promoter into a LUC vector. We found that all PpyCBFs had transcriptional activities with the $6 \mathrm{X}$ $\mathrm{C}$-repeat binding sites, with the ancestral CBF (PpyCBF3) showing the strongest interaction with these binding sites (Figure S5).

To further investigate possible transcriptional regulatory linkages involved in pear abiotic stress pathways, dual luciferase (in vitro) and $\mathrm{Y} 1 \mathrm{H}$ (in vivo) assays were performed with $P p y C B F$ and PpyCOR promoters. The dual luciferase assays revealed that PpyCBFs 1-6, PpyCBFs 1,2,4,5, PpyCBFs 1-4, and PpyCBF2 could significantly transactivate the promoters of PpyCOR47, PpyCOR15A, PpyRD29A, and PpyKIN, respectively. Clade II PpyCBFs had high transcriptional activities with PpyCOR47, 15A, and RD29A, while clade I and III PpyCBFs had little interaction with PpyRD29A (Figure 5a). In view of these results, $\mathrm{Y} 1 \mathrm{H}$ assays were performed between $P p y C B F$ genes and $P p y C O R$ promoters. The $\mathrm{Y} 1 \mathrm{H}$ results validated the direct interactions of PpyCBFs 2,4,5 with PpyCOR47, PpyCBFs 2 and 5 with PpyCOR15A, and PpyCBFs 2 and 4 with PpyRD29A promoters, while no interactions were detected between PpyKIN-PpyCBFs. Interestingly, the ancestral CBF did not show any physical interaction with stress-responsive genes, while P PyCBF2 was found to be the most active transcriptional regulator during abiotic stress signaling (Figure $5 b$ ). 


\section{a}

b

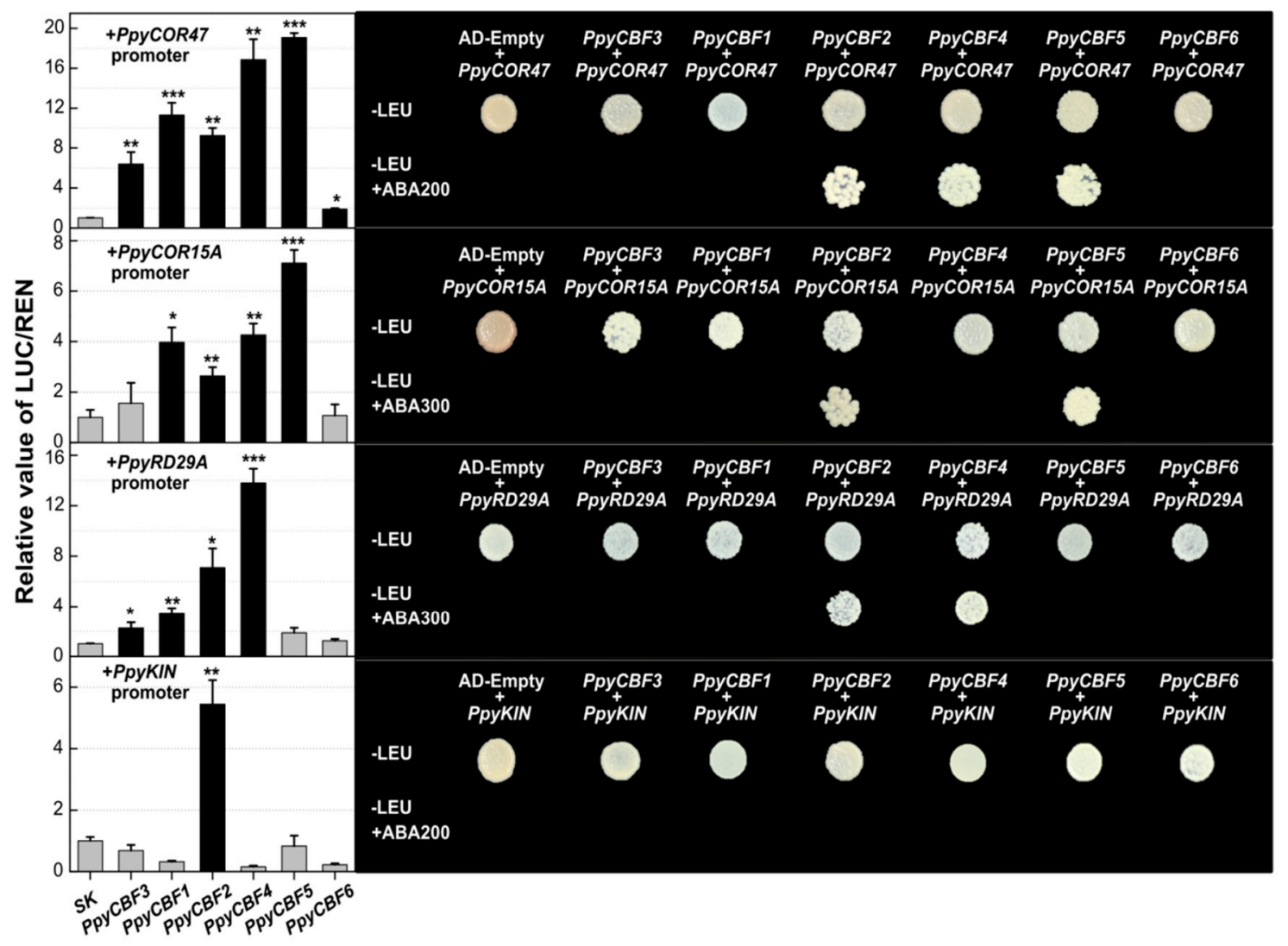

Figure 5. In vivo and in vitro regulations of PpyCBFs on the promoters of stress-related genes. (a) Dual luciferase assay to check the in vitro regulations. The ratio of firefly luciferase/renilla luciferase (LUC/REN) of the empty vector (pGreenII 0029 62-SK) plus promoter was used as calibrator (set as 1). Three independent experiments were done to verify the results. Error bars show SEs with at least four biological replicates, while asterisks show significant differences of genes SK with empty SK $\left({ }^{*} p<0.05\right.$, ** $p<0.01,{ }^{* * *} p<0.001$ ). (b) $\mathrm{Y} 1 \mathrm{H}$ assay shows in vivo binding of PpyCBFs on PpyCOR promoters. Synthetic dropout (SD) medium without Leu and supplemented with 200 and $300 \mathrm{ng} \mathrm{mL}-1$ ABA was used. Yeast grew on ABA-supplemented plates, indicating the possible direct interactions.

\subsection{PpyCBFs Can Also Bind at the TCGAC Binding Site in the PpyCOR15A Promoter}

The above findings indicate that $P p y C B F s$ have transcriptional activities with 6X CCGAC binding sites. According to an analysis of PpyCOR promoters, however, PpyCOR15A had no CRT binding site in its promoter region, but had high transcriptional activities with PpyCBFs (Table S3). To identify the unique PpyCBF binding site in the PpyCOR15A promoter, we therefore first divided the PpyCOR15A promoter into four fragments. We observed both in vitro and in vivo interactions of PpyCBFs with fragment 2 of PpyCOR15A (Figure 6b,c). We identified three possible CBF-binding sites in this region, CGACA, CCGA and TCCG, and mutated them into CTTTA, CTTT and GTTG, respectively (Figure 6a). Luciferase and $\mathrm{Y} 1 \mathrm{H}$ assays proved that the mutation at the CGACA binding site reduced the transcriptional activities and physical interactions of all PpyCBFs with the PpyCOR15A promoter present at -615 to $-610 \mathrm{bp}$ from the start codon. No effects on transcriptional regulation or direct interactions were observed at the second and third mutation sites. Hence, PpyCBFs can also bind to the TCGAC binding site, and the deletion of one cytosine from the CRT binding site did not influence its binding activity with the PpyCOR15A promoter in pears. 


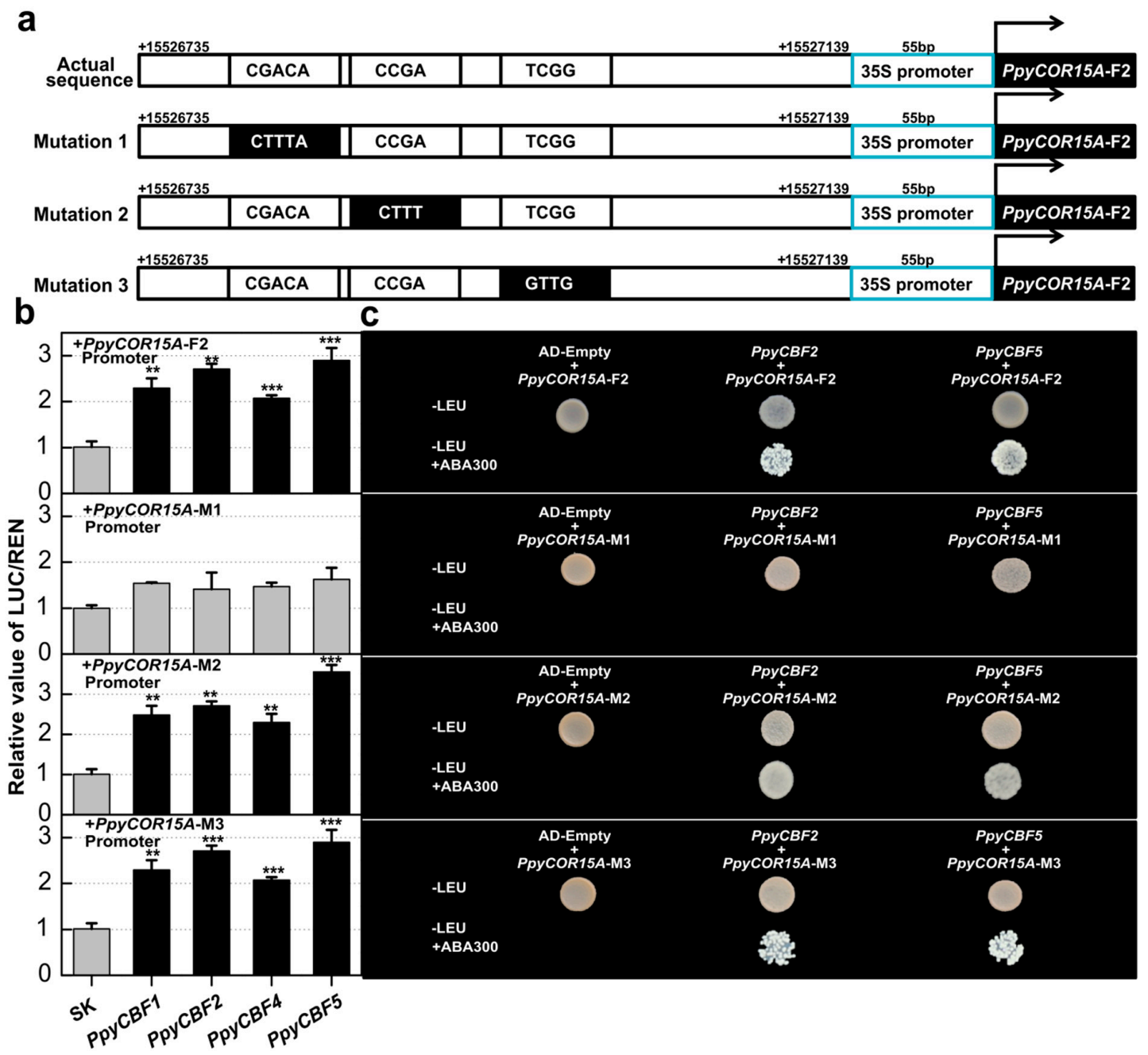

Figure 6. PpyCBFs can also bind at TCGAC binding site in the PpyCOR15A promoter. (a) Schematic diagrams of mutations at three different motif sites for PpyCOR15A promoters, indicated with mutation 1, 2, and 3. Possible CBF-binding sites in PpyCOR15A promoter are represented with white rectangles while mutations at these sites are represented by black rectangles. (b) Dual-luciferase assays were performed with actual and mutated promoters of the PpyCOR15A promoter. The ratio of LUC/REN of the empty vector (pGreenII 0029 62-SK) plus promoter was used as the calibrator (set as 1). Three independent experiments (with minimum four replicates) were performed to verify the results. Error bars show SEs with at least four biological replicates while asterisks show significant differences with empty SK $\left(* * p<0.01,{ }^{* * *} p<0.001\right)$. (c) $\mathrm{Y} 1 \mathrm{H}$ assay was performed to check physical interaction of PpyCBFs 2 and 5 with actual and mutated promoters of PpyCOR15A. Yeast grows on synthetic dropout without leucine but having Aureobasidin A 300 (SD/-leu + ABA300) indicating the possible direct interactions.

\section{Discussion}

In this study, we isolated 15 PpyCBF TFs from the pear genome. On the basis of sequence identity, phylogeny, conserved domain sequence (CDS) completeness, and scaffold position, however, only six PpyCBFs genes were selected for further study (Figure 1 and Table S1). Several CBF-specific domains, especially AP2, had strong conservations in plants, ultimately reflecting their high levels of identity $[1,4]$. This result explains why many identical amino acid residues and homologous groups were also found among CBFs of pears (Table S1) and other crop species, such as Arabidopsis, soybeans, apples, grapes, and different grasses $[9,10,14,37]$. Phylogenetic analysis provided evidence of independent evolution and three main PpyCBF clades/subtypes, while collinearity analyses uncovered two duplicated gene 
pairs (Figure 1 and Figure S1). The first clade not only contained CBFs from dicot and monocot crop species, but also the collinear gene PpyCBF3. The presence of PpyCBF3 in this first clade along with genes from both monocots and dicots, and the evolutionary relationship of this clade to the other two CBF clades suggested that PpyCBF3 might be the ancestral CBF from which all other CBFs were derived during whole-genome duplication in pears prior to their divergence from apples. This result is similar to soybeans, where the presence of orthologs from both dicot and monocot plants suggests that GmDREB1 clade/subtype 4 genes are the ancestral genes in the GmDREB1 family [14]. Rosaceous and Arabidopsis crop CBFs may have evolved completely independently of one another, as CBF regulation in woody plants appears to be more complex than that in herbaceous plants [11].

As mentioned above, $P p y C B F s$ were found to have different predicted functions than those of $A t C B F s$, which was corroborated by abiotic stress and bud endodormancy experiments that revealed that PpyCBFs 1-6 were not only induced by low temperature, salt, and drought stresses, but also by exogenous and endogenous ABA (Figures $2 \mathrm{a}$ and 3 ). The predicted functions and expressions of these PpyCBFs were similar to those of MbDREB1 in apples [15], PaDREB1 in sweet cherries [38], $B r C B F$ in non-heading Chinese cabbages [39], and VviDREB1 in cowberries [40] during abiotic stress, but they were dissimilar to AtCBFs 1-3 in Arabidopsis, which is only low-temperature responsive [10]. A proposed explanation for these expression changes is that cold, drought, and high salinity all cause osmotic stress [5]. In Japanese pears during bud endodormancy, we observed that the expressions of CBF/DREB4, DREB1E, DREB2, DREB2A, and DREB2D first peaked on December 24 and then suddenly declined on January 8, with a second expression peak on January 20 in both 'TH3' and 'Hengshani' cultivars [41]. We hypothesized that the first peak was low-temperature-responsive, while the second was ABA-responsive. To confirm in vivo functions of $P$ PyCBFs in plants, we ectopically expressed two PpyCBF genes, PpyCBF2 and PpyCBF3, in Arabidopsis. We found that plants of the two exogenous PpyCBF-ox Arabidopsis lines had higher resistance to low temperature $\left(10^{\circ} \mathrm{C}\right)$, salt $(50 \mathrm{mM})$, and drought $(10 \%)$ stresses than the wild type (Figure $4 a)$, similar to results in transgenic plants overexpressing DREB1s from apples, soybeans, grapes, and cabbages [9,14,15,39]. Interestingly, overexpression of PpyCBFs did not cause a dwarf phenotype in transgenic Arabidopsis grown on Murashige-Skoog (MS) medium (Figure S4), an outcome in agreement with observations from overexpression of MbDREB1 genes in Arabidopsis [15]. One notable feature of low-temperature stress and CBF overexpression is that both cause marked growth retardation resulting from the promotion of GA catabolism by two CBF-regulated isoforms (GA2ox3 and GA2ox6) and subsequent accumulation of DELLA proteins [42]. Some evidence suggests that at least a few CBF paralogs have evolved to execute different functions [9], which would explain the differential responses of $P p y C B F$ paralogs to various stresses observed in our study (Figure 2a). In particular, $P p y C B F s$ from clade II were not only more cold-responsive during abiotic stress and bud endodormancy, but they also exhibited higher resistance in overexpressing Arabidopsis to cold stress compared with salt and drought stresses. In contrast, clade I and III CBFs were highly salt- and drought-responsive and were more resistant in transgenic Arabidopsis to these stresses (Figures 2 and 3). This situation is similar to soybeans, where the expressions of GmDREB1 genes assigned to phylogenetic subtypes 1 and 2 were found to be induced by low-temperature, salinity, drought, and heat stresses, whereas those of subtype 4 were only induced by low temperature and salt [14].

The expression patterns of CBFs and CORs in pear are similar to those in other plant species [34]. Our qRT-PCR analysis revealed that PpyCOR expressions were increased not only by cold, salt, and drought stresses, but also by endogenous and exogenous ABA (Figure $2 b$ ). This result is unsurprising, as CBF-induced tolerance to cold, salt, drought, and ABA has been repeatedly correlated with increased expressions of COR genes [9]. Significantly higher amounts of PpyCOR15a and PpyCOR47 transcripts were detected during abiotic stress, however, the reason why the expressions of PpyRD29A and PpyKIN did not follow the same trend as other COR genes is unclear. We note that specific information on all COR genes in pears are still limited. In regard to the effect of PpyCBFs on endogenous ABA-dependent and -independent genes, we observed significantly higher expressions of these genes 
under normal, unstressed conditions in PpyCBF2-ox and PpyCBF3-ox lines than in the wild type (Figure $4 \mathrm{~d}$ ). These findings suggest that PPyCBF2 and PpyCBF3 participate in both ABA-dependent and -independent pathways during abiotic stress signaling. Similar findings have also been reported for apples, grapes, and potatoes, where overexpressed MbDREB1, $V v C B F$, and ScCBF1 significantly increase the expressions of ABA-independent (AtCOR15a, AtRD29A, AtCOR6.6, and AtCOR47) and ABA-dependent (AtRD29B, AtRAB18, AtABI1, and AtABI2) genes during normal conditions $[9,15]$. Interestingly, the expressions of all stress-responsive genes during abiotic stress conditions were significantly lower in overexpressing lines than the wild type, as the overexpressing lines had more resistance than the wild type because of the endogenous activation of AtCOR genes (Figure 4d).

Upon further investigation of transcriptional regulatory pathways of $P p y C B F s$, we uncovered their central role during abiotic stress signaling in pears (Figure 5 and Table 1). The results of our luciferase and $\mathrm{Y} 1 \mathrm{H}$ assays indicated the existence of at least two main types of transcriptional interactions associated with CBF clades. In other words, all clade CBFs (except PpyCBF6) had interactions with PpyCOR47 and 15A, while clade II PpyCBFs had a stronger association with PpyRD29A compared with clades I and III. PpyCBFs were involved in the same CBF-COR cascades during abiotic stresses that are conserved in multiple plant species such as Arabidopsis and Brachypodium, with AtCBF1-3 and $B d C B F 1$ showing interactions with COR genes by binding CRT/DRE (CCGAC) elements [34,37]. We also observed high transcriptional activities of all PpyCBFs with 6XCRT/DRE (CCGAC) binding sites. An analysis of PpyCOR gene promoters uncovered no CCGAC binding sites in the promoters of PpyCOR15A, PpyKIN, or PpyRD29A (Table S3), but we detected their strong in vivo and in vitro interactions with PpyCBFs. By mutating the CGAC binding site in PpyCOR15A, we were able to determine that $P p y C B F s$ can also bind to the TCGAC binding site (Figure 6). In our previous study, we found that $P p C B F 2$ can also bind to the CCGA binding site in the PpCBF4 promoter [22], which indicates that CGA is the actual core of the CBF binding site in pears.

To investigate the underlying mechanism of transcriptional regulation of PpyCBF expression by abiotic stress and ABA treatments, we examined the promoter regions of all PpyCBFs (Table 1). We found that PpyCBF expressions during abiotic stress are regulated by CRT/DRE, GT-1-like box, ICE1-like, NAC, and I BOX TFs, whereas during ABA treatment, ABRE and G-box1 TFs are involved. A bZIP transcription factor specifically recognizes G-box1 in promoters of ABA-responsive genes [43]. The absence of G-box1 cis elements and the presence of ABRE cis elements in PpyCBF3 and PpyCBF5 indicates that these genes are only regulated by the ABI3/VP1 cascade. In contrast, clade II PpyCBFs are regulated by both b-ZIP and ABI3 TFs, which explains why the expressions of clade II CBFs during ABA stress were relatively higher than those of PpyCBF3 and PpyCBF5 (Figure 2a). NAC TFs in pears are highly abiotic-stress responsive [44]. ICE-1 encoding a MYC-like basic helix-loop-helix protein that binds to Myc recognition sequences [33] and transcriptional induction of PpCBFs by PpICE1s have already been observed in pears [22]. DREB1 genes are also negatively regulated by MYB15, an R2R3-type MYB transcription factor in Arabidopsis [7]. In both Arabidopsis and soybeans, a bZip TF recognizes GT-1-like boxes and plays a role in salt- and pathogen-induced gene expression [45]. MIKC cis elements in PpyCBFs also display a dormancy response, as the CBF-DAM regulon aids pear adaptation through bud endodormancy [22]. Given the above mentioned results, the relatively high abundance of PpyCBFs in the face of abiotic stress as well as exogenous and endogenous ABA, the induction of ABA-dependent and -independent genes in overexpressed Arabidopsis under control conditions, and the in vivo and in vitro interactions of PpyCBFs with PpyCORs and the presence of both stress- and ABA-related cis elements in their promoters.

\section{Materials and Methods}

\subsection{Identification and Characterization of PpyCBFs}

Protein sequences of PpyCBF subfamily members and PpyCORs were retrieved from the Pear Genome Project database (http://peargenome.njau.edu.cn/), while two databases were used 
to obtain Malus (Md), Prunus (Ppe), Fragaria (Fv), and Vitis (Vv) CBFs: The Genome Database for Rosaceae (GDR; http://www.rosaceae.org/) and the Plant Transcription Factor database (Plant TFDB v4.0; http://planttfdb.cbi.pku.edu.cn/). AtCBFs were downloaded from the Arabidopsis Information Resource (https://www.arabidopsis.org/). Collinear blocks of PpyCBFs and whole genomes within species were identified in MCScanX with default settings and an $E$-value $\leq 1 \times 10^{-10}$. After aligning all sequences in ClustalX, the resulting identity matrix was checked using BioEdit software. Phylogenetic analysis of PpyCBFs and CBFs of other crop species was performed by the neighbor-joining method with 1000 bootstrap replicates in MEGA v7.0. Gene structure and motif analyses were carried out using Gene Structure Display Server v2.0 (http://gsds.cbi.pku.edu.cn/) and MEME v5.0.4 (http://meme-suite.org/tools/meme) tools with default parameters. The PlantPan2.0 (http://plantpan2.itps.ncku.edu.tw/) database with 2000 nucleotides was used for promoter analysis.

\subsection{Plant Materials and Abiotic Stress Treatments}

For abiotic stress experiments, vegetative buds of Asian pear cultivar 'Dangshan Suli' were collected before bud break in March 2018. After collection, buds were washed, sterilized, and then grown in half-strength MS medium to generate pear seedlings. Seedlings of a uniform size with six to eight leaves were randomly selected for abiotic stress treatments. For the low temperature treatment, seedlings in MS medium were exposed to $4{ }^{\circ} \mathrm{C}$, while drought and salt stress treatments were carried out by respectively adding $200 \mathrm{mM} \mathrm{NaCl}$ and $15 \%$ PEG6000to half-strength MS medium. Samples were collected with three replicates after $0,6,12,24$, and $48 \mathrm{~h}$ of treatment. For ABA stress treatments, wild-type pear calli were placed in half-strength MS medium containing $100 \mu \mathrm{M} \mathrm{ABA}$ (stressed) or $100 \mu \mathrm{M}$ absolute ethanol (Mock), and sampling was carried out with three replicates of each treatment group after $0,3,6,12,24$, and $48 \mathrm{~h}$. Following the abiotic stress treatments, each sample was immediately frozen in liquid nitrogen and stored at $-80^{\circ} \mathrm{C}$. Plant materials and methods for study of bud endodormancy in pears were the same as those of a previously published study [44].

\subsection{Analysis of Stress Tolerance of Transgenic Plants}

After amplification, PpyCBF2 and PpyCBF3 coding sequences were cloned into a pCAMBIA 1301 vector to generate 35S::PpyCBFs constructs. The recombinant plasmids were inserted into Agrobacterium EHA105 cells and then transformed into flowering Arabidopsis thaliana plants by the floral dip method. After 7 days, the floral dip procedure was repeated. Following seed collection, the transgenic Arabidopsis plants were screened on MS medium containing $1 \mu \mathrm{g} \mathrm{mL} \mathrm{L}^{-1}$ of the antibiotic hygromycin. Putative transformants among the $\mathrm{T}_{1}$ progeny, confirmed by RT-PCR using PpyCBF2and PpyCBF3-ORF-F/R primers, were regrown using the same procedure to obtain $\mathrm{T}_{3}$ progeny. The line of $\mathrm{T}_{3}$ plants with the highest PpyCBF2 and PpyCBF3 abundances was selected and grown to generate $\mathrm{T}_{4}$ progeny, which were used to assess in vivo abiotic stress tolerance. For this assessment, seeds of wild-type and overexpressed lines were germinated on MS medium for 14 days, and their seedlings were then grown for 5 days on vertical plates containing MS medium supplemented with either $50 \mathrm{mM}$ $\mathrm{NaCl}$ (to assess salt tolerance) or 10\% PEG (to assess drought tolerance). As a control, another set of seedlings were grown on MS medium with no supplement. To assess cold tolerance, seedlings on MS plates were exposed to $10{ }^{\circ} \mathrm{C}$ for 21 days. After abiotic stress treatments, all seedlings were grown under normal conditions on MS medium for 5 days to check their recovery rate. ImageJ v1.8.0 software was used to measure root lengths of wild-type and overexpressed lines under normal and abiotic stress conditions.

\subsection{Histochemical Analysis of $\mathrm{H}_{2} \mathrm{O}_{2}$ and $\mathrm{O}_{2}{ }^{\bullet-}$}

For histochemical analysis of $\mathrm{H}_{2} \mathrm{O}_{2}$ and $\mathrm{O} 2^{\bullet-}$, fresh diaminobenzidine (DAB) and nitroblue tetrazolium (NBT) solutions were prepared following a method reported previously [46]. Plant leaves were immersed in DAB and NBT solutions and incubated overnight at room temperature in darkness, the latter achieved by wrapping in aluminum foil. To remove chlorophyll for proper visualization, 
the leaves were bleached in absolute ethanol for $10 \mathrm{~min}$ at $95^{\circ} \mathrm{C}$ in a water bath. Photographs of stained samples were taken using a Leica DMLB fluorescence microscope, where brown and blue spots respectively indicated the presence of $\mathrm{H}_{2} \mathrm{O}_{2}$ and $\mathrm{O}_{2}{ }^{\bullet-}$ in situ.

\subsection{RNA Extraction and cDNA Synthesis}

Total RNA was extracted from three biological replicates using a modified cetyltrimethylammonium bromide method as described in our previous study [47]. cDNA was then synthesized from $4 \mu \mathrm{g}$ of DNA-free RNA using an iScript cDNA Synthesis kit (Bio-Rad, Foster, CA, USA) following the manufacturer's instructions. Ten-fold diluted cDNA was used as a template for qRT-PCR analysis.

\section{6. $q R T-P C R$ Analysis}

qRT-PCR amplifications were performed in $15 \mu \mathrm{L}$ reaction volumes composed of $7.5 \mu \mathrm{L}$ SYBR Premix Ex Taq (TliRNaseH Plus, Takara Biotechnology (Dalian) Co., Ltd. Dalian, China), $1 \mu \mathrm{L}$ cDNA, $0.5 \mu \mathrm{L}$ each of forward and reverse primers, and $5.5 \mu \mathrm{L}$ RNase-free water. The amplifications were carried out on a CFX Connect real-time PCR system (Bio-Rad, Hercules, CA, USA) according to the following protocol: $95{ }^{\circ} \mathrm{C}$ for $30 \mathrm{~s}$, followed by 40 cycles of $95{ }^{\circ} \mathrm{C}$ for $5 \mathrm{~s}$ and $60{ }^{\circ} \mathrm{C}$ for $20 \mathrm{~s}$. Melting curves were used to confirm the specificity of the qRT-PCR primers. Relative gene transcript levels were determined using the $2^{-\Delta \Delta \mathrm{Ct}}$ method and normalized against PpyActin (JN684184).

\subsection{Site-Directed Mutagenesis of Gene Promoters}

To check possible binding sites of PpyCBFs in PpyCOR promoters, the predicted sites were altered by directed mutagenesis. Motif mutations were carried out using a mutagenesis system after designing specific primers for possible binding sites. Transactivation effects of PpyCBFs on mutated promoters were further examined using dual luciferase and $\mathrm{Y} 1 \mathrm{H}$ assays.

\subsection{Transient Expression and Luciferase Measurement}

A dual luciferase assay was used to detect in vivo transactivation effects of transcription factors. Full-length PpyCBF and PpyCOR promoters (2000 nucleotides) were inserted into pGreenII 0029 62-SK and pGreenII 0800-LUC vectors, respectively. The dual luciferase assay was carried out with Nicotiana benthamiana leaves according to our previously described protocol [22]. Three independent experiments with a minimum of four replicates were performed to verify the results.

\subsection{Yeast One-Hybrid Assay}

Y1H assays were conducted using a Matchmaker Gold Yeast One-Hybrid System kit (Clontech, Takara, Japan) according to the instructions in the user manual. Subsequent analyses were completed as previously described [48].

\subsection{Statistical Analysis}

Experiments were set up according to a completely randomized design. Analysis of variance followed by Duncan's multiple range test was used to test the overall significance of differences among treatments $(p<0.05)$. Significant differences between treatments were assessed by Student's $t$-test at $p<0.05, p<0.01$, and $p<0.001$. All data were analyzed in SPSS v25 (SPSS Inc., Chicago, IL, USA).

\section{Conclusions}

We identified six PpyCBF homologues (PpCBF1-6) encoding potential transcription factors in Asian pear. All PpyCBF members accentuated during different abiotic stresses and endo and exogenous ABA. II clade PpyCBFs were not only more low temperature (LT) and ABA responsive but also enhanced LT stress tolerance in overexpressed Arabidopsis as compared to I and III clades PpyCBFs. Ectopic expressions of PpyCBF2 and PpyCBF3 in Arabidopsis also increased the expressions of endogenous 
ABA dependent and independent genes during normal conditions. A conversed CBF-COR regulatory cascade was also observed in pear. We conclude that PpyCBFs may follow both ABA-dependent and -independent stress signaling pathways during abiotic stress in pears. PpyCBF transcription factors may thus act redundantly during abiotic stress through ABA-dependent and -independent pathways. The results of our investigation, the first to differentiate the functions of the complete CBF subfamily in any rosaceous crop species, should have an important influence on the study of stress in woody species and may be applicable for the genetic engineering of different functions of transcription factors in other plant species.

Supplementary Materials: Supplementary materials can be found at http://www.mdpi.com/1422-0067/20/9/2074/s1.

Author Contributions: S.B. and Y.T. perceived and planned the study and M.A. and J.L. performed most of the experiments and analyses. M.A. and Q.Y. collected the samples and extracted total RNAs for qPCR. J.L. and W.J. helped in luciferase and $\mathrm{Y} 1 \mathrm{H}$ assays, and data arrangements. M.A., S.B., and Y.T. wrote the manuscript. All authors read and approved the final manuscript.

Funding: This work was supported by the National Key Research and Developmental Program of China (2018YFD1000104) to S.B., National Natural Science Foundation of China (31501736) to S.B., and the Earmarked Fund for China Agriculture Research System (CARS-28) to Y.T.

Acknowledgments: We thank the Dangshan Suli Germplasm Resources Center for providing plant materials. We also say special thanks to Muhammad Ali Raza for valuable efforts and instructions in growing of transgenic Arabidopsis.

Conflicts of Interest: The authors declare no conflict of interest.

\section{Abbreviations}

$\begin{array}{ll}\text { CORs } & \text { Cold Regulons } \\ \text { HMM } & \text { Hidden Markov Model } \\ \text { MEGA } & \text { Molecular Evolutionary Genetics Analysis } \\ \text { TF } & \text { Transcription factor } \\ \text { Y1H } & \text { Yeast one hybrid } \\ \text { SnRK2 } & \text { Snf1-Related kinase 2 } \\ \text { CTAB } & \text { Cetyltrimethyl Ammonium Bromide }\end{array}$

\section{References}

1. Nakano, T.; Suzuki, K.; Fujimura, T.; Shinshi, H. Genome-wide analysis of the ERF gene family in Arabidopsis and rice. Plant Physiol. 2006, 140, 411-432. [CrossRef] [PubMed]

2. Licausi, F.; Ohme Takagi, M.; Perata, P. APETALA 2/Ethylene Responsive Factor (AP 2/ERF) transcription factors: Mediators of stress responses and developmental programs. New Phytol. 2013, 199, 639-649. [CrossRef]

3. Jaglo, K.R.; Kleff, S.; Amundsen, K.L.; Zhang, X.; Haake, V.; Zhang, J.Z.; Deits, T.; Thomashow, M.F. Components of the Arabidopsis C-repeat/dehydration-responsive element binding factor cold-response pathway are conserved in Brassica napus and other plant species. Plant Physiol. 2001, 127, 910-917. [CrossRef]

4. Sakuma, Y.; Liu, Q.; Dubouzet, J.G.; Abe, H.; Shinozaki, K.; Yamaguchi-Shinozaki, K. DNA-binding specificity of the ERF/AP2 domain of Arabidopsis DREBs, transcription factors involved in dehydration-and cold-inducible gene expression. Biochem. Biophys. Res. Commun. 2002, 290, 998-1009. [CrossRef] [PubMed]

5. Thomashow, M.F. Molecular basis of plant cold acclimation: Insights gained from studying the CBF cold response pathway. Plant Physiol. 2010, 154, 571-577. [CrossRef]

6. Welling, A.; Palva, E.T. Involvement of CBF transcription factors in winter hardiness in birch. Plant Physiol. 2008, 147, 1199-1211. [CrossRef] [PubMed]

7. Agarwal, P.K.; Agarwal, P.; Reddy, M.; Sopory, S.K. Role of DREB transcription factors in abiotic and biotic stress tolerance in plants. Plant Cell Rep. 2006, 25, 1263-1274. [CrossRef]

8. Mizoi, J.; Shinozaki, K.; Yamaguchi Shinozaki, K. AP2/ERF family transcription factors in plant abiotic stress responses. Biochim. Biophys. Acta 2012, 1819, 86-96. [CrossRef]

9. Siddiqua, M.; Nassuth, A. Vitis CBF1 and Vitis CBF4 differ in their effect on Arabidopsis abiotic stress tolerance, development and gene expression. Plant Cell Environ. 2011, 34, 1345-1359. [CrossRef] [PubMed] 
10. Novillo, F.; Alonso, J.M.; Ecker, J.R.; Salinas, J. CBF2/DREB1C is a negative regulator of CBF1/DREB1B and CBF3/DREB1A expression and plays a central role in stress tolerance in Arabidopsis. Proc. Natl. Acad. Sci. USA 2004, 101, 3985-3990. [CrossRef]

11. Benedict, C.; Skinner, J.S.; Meng, R.; Chang, Y.; Bhalerao, R.; Huner, N.P.; Finn, C.E.; Chen, T.H.; Hurry, V. The CBF1-dependent low temperature signalling pathway, regulon and increase in freeze tolerance are conserved in Populus spp. Plant Cell Environ. 2006, 29, 1259-1272. [CrossRef] [PubMed]

12. Xiao, H.; Siddiqua, M.; Braybrook, S.; Nassuth, A. Three grape CBF/DREB1 genes respond to low temperature, drought and abscisic acid. Plant Cell Environ. 2006, 29, 1410-1421. [CrossRef] [PubMed]

13. Xiao, H.; Tattersall, E.A.; Siddiqua, M.K.; Cramer, G.R.; Nassuth, A. CBF4 is a unique member of the CBF transcription factor family of Vitis vinifera and Vitis riparia. Plant Cell Environ. 2008, 31, 1-10. [CrossRef]

14. Kidokoro, S.; Watanabe, K.; Ohori, T.; Moriwaki, T.; Maruyama, K.; Mizoi, J.; Myint Phyu Sin Htwe, N.; Fujita, Y.; Sekita, S.; Shinozaki, K. Soybean DREB 1/CBF type transcription factors function in heat and drought as well as cold stress responsive gene expression. Plant J. 2015, 81, 505-518. [CrossRef] [PubMed]

15. Yang, W.; Liu, X.D.; Chi, X.J.; Wu, C.A.; Li, Y.Z.; Song, L.L.; Liu, X.M.; Wang, Y.F.; Wang, F.W.; Zhang, C. Dwarf apple MbDREB1 enhances plant tolerance to low temperature, drought, and salt stress via both ABA-dependent and ABA-independent pathways. Planta 2011, 233, 219-229. [CrossRef] [PubMed]

16. Magome, H.; Yamaguchi, S.; Hanada, A.; Kamiya, Y.; Oda, K. dwarf and delayed-flowering 1, a novel Arabidopsis mutant deficient in gibberellin biosynthesis because of overexpression of a putative AP2 transcription factor. Plant J. 2004, 37, 720-729. [CrossRef]

17. Haake, V.; Cook, D.; Riechmann, J.; Pineda, O.; Thomashow, M.F.; Zhang, J.Z. Transcription factor CBF4 is a regulator of drought adaptation in Arabidopsis. Plant Physiol. 2002, 130, 639-648. [CrossRef]

18. Liu, Q.; Kasuga, M.; Sakuma, Y.; Abe, H.; Miura, S.; Yamaguchi Shinozaki, K.; Shinozaki, K. Two transcription factors, DREB1 and DREB2, with an EREBP/AP2 DNA binding domain separate two cellular signal transduction pathways in drought-and low-temperature-responsive gene expression, respectively, in Arabidopsis. Plant Cell 1998, 10, 1391-1406. [CrossRef] [PubMed]

19. Zhu, J.; Dong, C.H.; Zhu, J.K. Interplay between cold-responsive gene regulation, metabolism and RNA processing during plant cold acclimation. Curr. Opin. Plant Biol. 2007, 10, 290-295. [CrossRef]

20. Norén, L.; Kindgren, P.; Stachula, P.; Rühl, M.; Eriksson, M.E.; Hurry, V.; Strand, Å. Circadian and plastid signaling pathways are integrated to ensure correct expression of the $C B F$ and $C O R$ genes during photoperiodic growth. Plant Physiol. 2016, 171, 1392-1406.

21. Rubio, S.; Noriega, X.; Pérez, F.J. Abscisic acid (ABA) and low temperatures synergistically increase the expression of CBF/DREB1 transcription factors and cold-hardiness in grapevine dormant buds. Ann. Bot. 2018, 20, 1-9. [CrossRef]

22. Li, J.; Yan, X.; Yang, Q.; Ma, Y.; Yang, B.; Tian, J.; Teng, Y.; Bai, S. PpCBFs selectively regulate PpDAMs and contribute to the pear bud endodormancy process. Plant Mol. Biol. 2019, 1-12. [CrossRef]

23. Li, J.; Xu, Y.; Niu, Q.; He, L.; Teng, Y.; Bai, S. Abscisic Acid (ABA) promotes the induction and maintenance of Pear (Pyrus pyrifolia White Pear Group) flower bud endodormancy. Int. J. Mol. Sci. 2018, 19, 310. [CrossRef]

24. Xiong, L.; Wang, R.G.; Mao, G.; Koczan, J.M. Identification of drought tolerance determinants by genetic analysis of root response to drought stress and abscisic acid. Plant Physiol. 2006, 142, 1065-1074. [CrossRef] [PubMed]

25. Hetherington, A.M. Guard cell signaling. Cell 2001, 107, 711-714. [CrossRef]

26. Niu, Q.; Li, J.; Cai, D.; Qian, M.; Jia, H.; Bai, S.; Hussain, S.; Liu, G.; Teng, Y.; Zheng, X. Dormancy-associated MADS-box genes and microRNAs jointly control dormancy transition in pear (Pyrus pyrifolia white pear group) flower bud. J. Exp. Bot. 2015, 67, 239-257. [CrossRef] [PubMed]

27. Xie, Y.; Chen, P.; Yan, Y.; Bao, C.; Li, X.; Wang, L.; Shen, X.; Li, H.; Liu, X.; Niu, C. An atypical R2R3 MYB transcription factor increases cold hardiness by CBF dependent and CBF independent pathways in apple. New Phytologist. 2018, 218, 201-218. [CrossRef] [PubMed]

28. An, J.; Li, R.; Qu, F.; You, C.; Wang, X.; Hao, Y. An apple NAC transcription factor negatively regulates cold tolerance via CBF-dependent pathway. J. Plant Physiol. 2018, 221, 74-80. [CrossRef]

29. Jin, C.; Li, K.Q.; Xu, X.Y.; Zhang, H.P.; Chen, H.X.; Chen, Y.H.; Hao, J.; Wang, Y.; Huang, X.S.; Zhang, S.L. A novel NAC transcription factor, PbeNAC1, of Pyrus betulifolia confers cold and drought tolerance via Interacting with PbeDREBs and activating the expression of stress-responsive genes. Front. Plant Sci. 2017, 8, 1049. [CrossRef] 
30. Ma, Y.; Szostkiewicz, I.; Korte, A.; Moes, D.; Yang, Y.; Christmann, A.; Grill, E. Regulators of PP2C phosphatase activity function as abscisic acid sensors. Science 2009, 324, 1064-1068. [CrossRef]

31. Park, S.Y.; Fung, P.; Nishimura, N.; Jensen, D.R.; Fujii, H.; Zhao, Y.; Lumba, S.; Santiago, J.; Rodrigues, A.; Tsz fung, F.C. Abscisic acid inhibits type 2C protein phosphatases via the PYR/PYL family of START proteins. Science 2009, 324, 1068-1071. [CrossRef] [PubMed]

32. Yoshida, T.; Fujita, Y.; Sayama, H.; Kidokoro, S.; Maruyama, K.; Mizoi, J.; Shinozaki, K.; Yamaguchi-Shinozaki, K. $A R E B 1, A R E B 2$, and $A B F 3$ are master transcription factors that cooperatively regulate ABRE-dependent ABA signaling involved in drought stress tolerance and require ABA for full activation. Plant J. 2010, 61, 672-685. [CrossRef]

33. Zhan, X.; Zhu, J.K.; Lang, Z. Increasing freezing tolerance: Kinase regulation of ICE1. Dev. Cell 2015, 32, 257-258. [CrossRef]

34. Zhou, M.; Shen, C.; Wu, L.; Tang, K.; Lin, J. CBF dependent signaling pathway: A key responder to low temperature stress in plants. Crit. Rev. Biotechnol. 2011, 31, 186-192. [CrossRef] [PubMed]

35. Teng, Y. The pear industry and research in China. Acta Hortic. 2011, 909, 161-170. [CrossRef]

36. Teng, Y. Advances in the research on phylogeny of the genus Pyrus and the origin of pear cultivars native to East Asia. J. Fruit Sci. 2017, 34, 370-378.

37. Ryu, J.Y.; Hong, S.Y.; Jo, S.H.; Woo, J.C.; Lee, S.; Park, C.M. Molecular and functional characterization of cold-responsive C-repeat binding factors from Brachypodium distachyon. BMC Plant Biol. 2014, 14, 15. [CrossRef] [PubMed]

38. Kitashiba, H.; Ishizaka, T.; Isuzugawa, K.; Nishimura, K.; Suzuki, T. Expression of a sweet cherry DREB1/CBF ortholog in Arabidopsis confers salt and freezing tolerance. J. Plant Physiol. 2004, 161, 1171-1176. [CrossRef] [PubMed]

39. Jiang, F.; Wang, F.; Wu, Z.; Li, Y.; Shi, G.; Hu, J.; Hou, X. Components of the Arabidopsis CBF cold-response pathway are conserved in non-heading Chinese cabbage. Plant Mol. Biol. Rep. 2011, 29, 525-532. [CrossRef]

40. Wang, Q.J.; Xu, K.Y.; Tong, Z.G.; Wang, S.H.; Gao, Z.H.; Zhang, J.Y.; Zong, C.W.; Qiao, Y.S.; Zhang, Z. Characterization of a new dehydration responsive element binding factor in central arctic cowberry. Plant Cell Tissue Organ Cult. 2010, 101, 211-219. [CrossRef]

41. Takemura, Y.; Kuroki, K.; Jiang, M.; Matsumoto, K.; Tamura, F. Identification of the expressed protein and the impact of change in ascorbate peroxidase activity related to endodormancy breaking in Pyrus pyrifolia. Plant Physiol. Biochem. 2015, 86, 121-129. [CrossRef] [PubMed]

42. Achard, P.; Renou, J.P.; Berthomé, R.; Harberd, N.P.; Genschik, P. Plant DELLAs restrain growth and promote survival of adversity by reducing the levels of reactive oxygen species. Curr. Biol. 2008, 18, 656-660. [CrossRef] [PubMed]

43. Uno, Y.; Furihata, T.; Abe, H.; Yoshida, R.; Shinozaki, K.; Yamaguchi Shinozaki, K. Arabidopsis basic leucine zipper transcription factors involved in an abscisic acid dependent signal transduction pathway under drought and high-salinity conditions. Proc. Natl. Acad. Sci. USA 2000, 97, 11632-11637. [CrossRef] [PubMed]

44. Ahmad, M.; Yan, X.; Li, J.; Yang, Q.; Jamil, W.; Teng, Y.; Bai, S. Genome wide identification and predicted functional analyses of NAC transcription factors in Asian pears. BMC Plant Biol. 2018, 18, 214. [CrossRef] [PubMed]

45. Park, H.C.; Kim, M.L.; Kang, Y.H.; Jeon, J.M.; Yoo, J.H.; Kim, M.C.; Park, C.Y.; Jeong, J.C.; Moon, B.C.; Lee, J.H. Pathogen-and $\mathrm{NaCl}$-induced expression of the SCaM-4 promoter is mediated in part by a GT-1 box that interacts with a GT-1-like transcription factor. Plant Physiol. 2004, 135, 2150-2161. [CrossRef] [PubMed]

46. Khan, A.R.; Wakeel, A.; Muhammad, N.; Liu, B.; Wu, M.; Liu, Y.; Ali, I.; Zaidi, S.H.R.; Azhar, W.; Song, G. Involvement of ethylene signaling in zinc oxide nanoparticle mediated biochemical changes in Arabidopsis thaliana leaves. Environ. Sci. Nano 2019, 6, 341-355. [CrossRef]

47. Yang, Q.; Niu, Q.; Li, J.; Zheng, X.; Ma, Y.; Bai, S.; Teng, Y. PpHB22, a member of HD-Zip proteins, activates PpDAM1 to regulate bud dormancy transition in 'Suli' pear (Pyrus pyrifolia White Pear Group). Plant Physiol. Biochem. 2018, 127, 355-365. [CrossRef]

48. Tao, R.; Bai, S.; Ni, J.; Yang, Q.; Zhao, Y.; Teng, Y. The blue light signal transduction pathway is involved in anthocyanin accumulation in 'Red Zaosu' pear. Planta 2018, 1-12. [CrossRef] [PubMed]

(C) 2019 by the authors. Licensee MDPI, Basel, Switzerland. This article is an open access article distributed under the terms and conditions of the Creative Commons Attribution (CC BY) license (http://creativecommons.org/licenses/by/4.0/). 